\title{
The Complex Kinematics of Galaxies in Hickson 67
}

\author{
D. Bettoni
}

Osservatorio Astronomico di Padova

L. M. Buson

Osservatorio Astronomico di Capodimonte, Napoli

\begin{abstract}
.
The kinematics of galaxies belonging to the Hickson compact group HCG67 are investigated. The latter consists of four galaxies, three of which (a, c, d) are embedded in a common envelope. The fourth galaxy (b) is a spiral that is detected both in radio and in IR wave-bands. Our observations show that the three galaxies in apparent interaction are probably caught during an ongoing merger process.
\end{abstract}

\section{Introduction}

The influence of the environment on the formation and the evolution of galaxies has been re-evaluated in recent years. This is giving rise to increasing interest about one of the most dense environments known: that of Compact Groups. The true nature of Compact Groups is still controversial. The conventional scenario foresees that the members of each group should interact and merge to form a single elliptical galaxy in few orbital periods (Barnes 1985). The short evolution time imposed by such a scenario would suggest that present-day groups attained only recently their high densities. However, recent observations put severe limits on this hypothesis. The low fraction of observed blue galaxies (Zepf et al. 1991) in groups implies a longer lifetime for these systems, for instance. Moreover, the luminosity function of compact galaxy groups (hereafter CGGs) does not resemble that of field ellipticals, of which they should be the progenitors (Mendes de Oliveira and Hickson, 1991; Rabaça and Sulentic 1991). Finally, Mamon (1992) goes so far as to suggest the alternative interpretation that some of the CGGs are actually due to the random alignment of galaxies in loose groups or poor clusters.

To better understand the true nature of CGGs it is therefore necessary to know at which level the supposed high spatial density of the groups has produced measurable effects on member galaxies. One of the most interesting cases is that of HCG16; in this group Mendes de Oliveira et al. (1998) observed that it is probably a compact group in formation through the merging of close objects in a dense environment. In this paper we present a similar case: HCG67. In this group at least three out of four member galaxies present signs of a possible interaction and merging into a more compact structure. 


\section{Observations}

Spectra of HCG67 galaxies were obtained in May 1991 with the ESO $1.52 \mathrm{~m}$ telescope equipped with the $\mathrm{B} \& \mathrm{C}$ spectrograph. Long slit spectra were taken along two position angles $(\mathrm{PA})$ : $\mathrm{PA}=33^{\circ}$ and $17^{\circ}$ and in two spectral regions. We used a 600 lines $\mathrm{mm}^{-1}$ grating (with a dispersion of $59 \AA \mathrm{mm}^{-1}$ at the second order) for the region $\lambda \lambda 4600-5900 \AA$ giving a resolution of $0.88 \AA /$ pixel and a 1200 lines $\mathrm{mm}^{-1}$ grating (with a dispersion of $60 \AA \mathrm{mm}^{-1}$ at the first order) for the region $\lambda \lambda 5700-6000 \AA$ giving a resolution of $1.14 \AA /$ pixel. The CCD was a Thomson $1024 \times 1024$ pixel chip.

The closeness of the galaxies $\mathbf{a}, \mathbf{c}$ and $\mathbf{d}$ allowed us to obtain spectra of at least two objects with each single exposure. At $P A=17^{\circ}$ the slit crossed galaxies a and $\mathbf{c}$ approximately along their apparent major axes. When oriented along $\mathrm{PA}=33^{\circ}$ the slit included the nuclei of both galaxies $\mathbf{c}$ and $\mathbf{d}$, while also crossing a region in the periphery of galaxy $\mathbf{a}$ (the red spectrum was taken only at $\mathrm{PA}=17^{\circ}$ ). All the spectra were reduced in a standard way by means of the IRAF package. On each night, spectra of slowly-rotating giant stars of spectral type from late$\mathrm{G}$ to early- $\mathrm{K}$ were also taken as templates for the Fourier Quotient reduction. The Fourier Quotient technique (Bertola et al. 1984) was applied to all spectra to obtain the stellar velocity curves and velocity dispersion profiles. The gas kinematics was analyzed by fitting Gaussian profiles to the emission lines.

\section{The Group}

HCG 67 (VV 135, NGC 5306) is composed of four galaxies (see the image in Hickson 1993). The brightest galaxy (a) is classified by Hickson (1982) as an elliptical galaxy (E1) and includes in its extended halo the galaxies c and d, a spiral (Scd) and a S0, respectively. The fourth Sc galaxy b has a red-shift very close to that of the other members of the group but is slightly isolated from the other three galaxies. In Figure 1 are shown all the rotation curves and the velocity dispersion profiles obtained for the first three galaxies. Open symbols represent data for gas and full symbols for stars. In the following we give a brief description of each galaxy and of its observed kinematical properties:

Galaxy a: the brightest member of the group, shows both kinematical and morphological peculiarities. Mendes de Oliveira and Hickson (1994) point out that this galaxy has a double nucleus. An R band image of HCG67 obtained with the $1.82 \mathrm{~m}$ Asiago telescope confirms the presence of two nuclei with the northern, less luminous nucleus $\sim 8$ arcsec apart from the main nucleus. The presence of the two nuclei is well evident also from our spectra. The SW component has a very high velocity dispersion $\sigma=370 \pm 31 \mathrm{kms}^{-1}$ and low rotation $V_{\max } \leq 30$ $\mathrm{km} / \mathrm{sec}$, while the NE component has a much lower velocity dispersion $\bar{\sigma}=$ $180 \pm 20 \mathrm{~km} \mathrm{~s}^{-1}$ and a higher rotation velocity $V_{\max } \sim 180 \mathrm{~km} \mathrm{~s}^{-1}$.

Galaxy b: is the only object of this group lacking kinematical information. Its morphology is clearly that of a spiral galaxy with a strong dust-lane across its disk (though no signatures of interaction can be recognized from its appearance). It is also an IR and radio source (Hickson et al. 1989, Menon 1995), hosting $10^{10} M_{\odot}$ of molecular gas (Leon et al. 1997). 


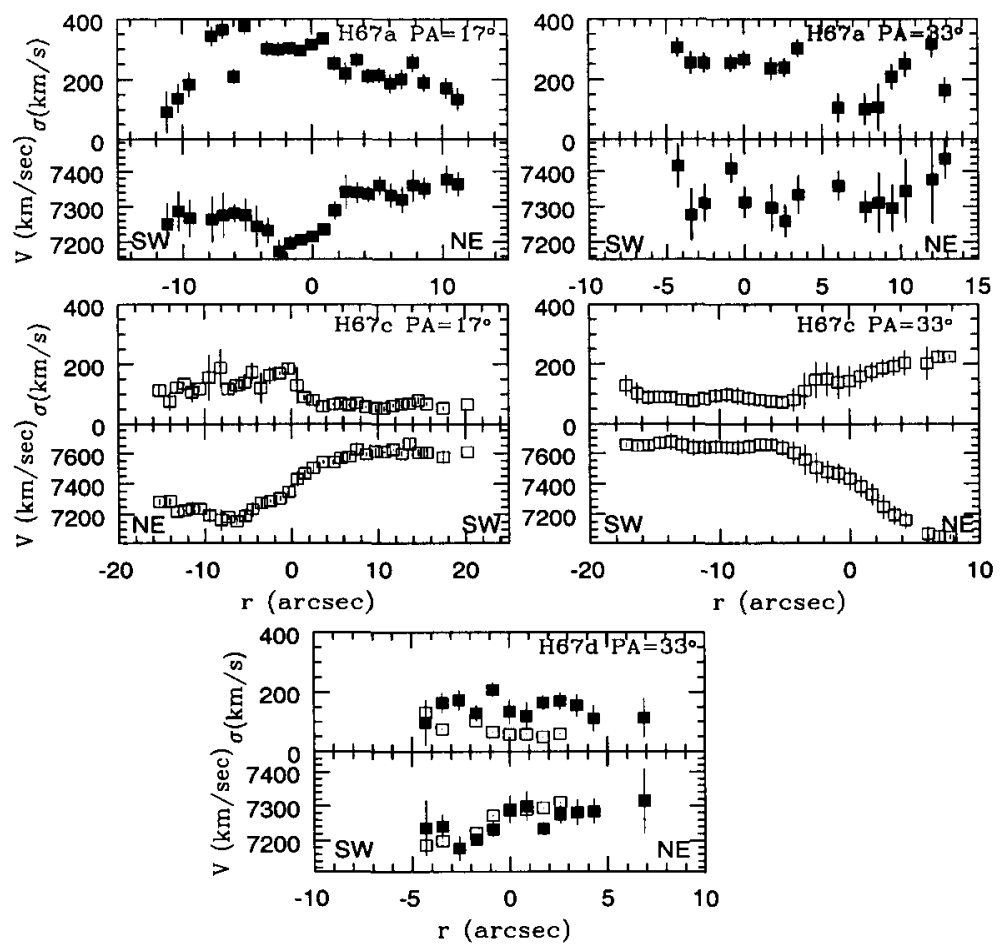

Figure 1. Velocity and velocity dispersion curves for galaxies HCG67a,b,c

Galaxy c: is a spiral and shows the typical emission lines of late-type galaxies. Its nuclear morphology is quite asymmetric in our image, presumably owing to the presence of a southern, fainter nucleus aligned along the apparent galaxy major axis, in the direction of the dominant galaxy a. The emission lines are very broad on the side opposite to galaxy a. Strangely enough, in this region a second sharp component in the emission lines is visible. This latter component, though being blue-shifted by $\Delta V \sim 490 \mathrm{~km} \mathrm{~s}^{-1}$, is also rotating and reaches a maximum velocity $\left(V_{\max } \sim 200 \mathrm{~km} \mathrm{~s}^{-1}\right)$ analogous to that of the main gaseous disk.

Galaxy d: In this S0 galaxy the stars and the gas corotate with a similar gradient and a $V_{\max } \sim 80 \mathrm{~km} \mathrm{~s}^{-1}$. The stellar velocity dispersion is almost constant at a value of $\sigma=151 \pm 36 \mathrm{~km} \mathrm{~s}^{-1}$.

\section{Discussion}

The brightest galaxy of the group (a) strongly resembles a Dumbell galaxy (e.g. UGC 2755 Balcells et al. 1995), though in our case the observed velocity difference between the two nuclei is quite low $\left(\sim 80 \mathrm{~km} \mathrm{~s}^{-1}\right)$. This might support 
the hypothesis that within few orbital periods the two nuclei will merge, possibly resulting in an the elliptical with a kinematically-independent stellar core. In this respect, it is noteworthy that the evolutionary time-scale of such a process could be surprisingly short and very close to the value of the crossing time in compact groups. The lack of gas in the dominant galaxy can be explained either if we suppose that it comes from two (or more) progenitors (whose remnants are the two nuclei) particularly gas-poor, or that the gas has formed stars very efficiently during the merging process.

Analogously, the double gas component observed in the spiral $\mathbf{c}$ might be the proof of a merging between two pre-existing galaxies. Alternatively, it could be the residual of tidal effects of an encounter with a dwarf, gas-rich object. The lower systemic velocity of the second component may be interpreted as gas falling toward the nucleus of the galaxy.

The gas observed in the S0 may also originate from a gravitational interaction, even if its kinematics is similar to that of the stellar component. The final direction of rotation in such a case depends on the mechanics of the collision and from the spin of the single, interacting galaxies. In this respect, the apparent normal kinematics of this galaxy d does not exclude a previous interaction with other members of group.

In summary, three out of four galaxies in the group HCG67 seem to share a common envelope and two of them show clear signatures of past merging. Our conclusion is that this group is in a transient configuration and may represent an intermediate stage of a merging event.

\section{References}

Balcells, M., Morganti, R., Oosterloo, T., Peréz-Fournon, I. González Serrano, J. I. 1995, A\&A, 302, 665 .

Bertola, F., Bettoni, D., Rusconi, L., Sedmak, G. 1984, AJ, 89, 356

Barnes, J. 1985, MNRAS, 215, 517

Hickson, P. 1982, ApJ, 255, 382

Hickson, P. 1993, Astrophys. Lett. Commun., 29, 1

Hickson, P., Menon, T. K., Palumbo, G. G. C., Persic, M. 1989, ApJ, 341,679

Leon, S., Combes, F., Menon, T. K. 1998, A\&A, 330, 37

Mamon, G. A. 1992,in Physics of Nearby Galaxies: Nature or Nurture?, ed. T. X6. Thuan, C. Balkowski \& Thran Thanh Van (12th Moriond Astrophysics Meeting)(Editions Frontiéres), p.367.

Mendes de Oliveira, C., Hickson, P. 1991, ApJ, 380, 30

Mendes de Oliveira, C., Plana, H, Amram, P., Bolte, M., Boulesteix, J. 1998, ApJ, 507, 691

Menon, T.K. 1995, MNRAS, 274, 845

Rabaça, C. R., Sulentic, J. W. 1991, BAAS, 23, 1338

Zepf, S. E., Whitmore, B. C., Levison, H. F. 1991, ApJ, 383, 524 